\title{
Impact of Spinal Correction Surgeries with Osteotomy and Pelvic Fixation in Patients with Kyphosis Due to Osteoporotic Vertebral Fractures
}

\author{
Tomohiko Hasegawa ${ }^{1}$, Hiroki Ushirozako ${ }^{1}$, Yu Yamato ${ }^{2}$, Go Yoshida ${ }^{1}$, Tatsuya Yasuda ${ }^{1}$, Tomohiro Banno $^{1}$, \\ Hideyuki Arima ${ }^{1}$, Shin $\mathrm{Oe}^{2}$, Tomohiro Yamada ${ }^{1}$, Koichiro Ide ${ }^{1}$, Yuh Watanabe ${ }^{1}$, Yukihiro Matsuyama ${ }^{1}$ \\ ${ }^{1}$ Department of Orthopedic Surgery, Hamamatsu University School of Medicine, Hamamatsu, Japan \\ ${ }^{2}$ Division of Geriatric Musculoskeletal Health, Department of Orthopedic Surgery, Hamamatsu University School of Medicine, Hamamatsu, Japan
}

Study Design: Combination of retrospective and prospective study.

Purpose: We aimed to compare the clinical outcomes between local fixation surgery and spinopelvic fixation surgery for the treatment of kyphosis secondary to osteoporotic vertebral fractures with spinopelvic malalignment.

Overview of Literature: The clinical characteristics of patients with rigid kyphosis due to osteoporotic vertebral fracture differ from that of middle-aged patients with vertebral fractures in terms of bone fragility and presence of spinopelvic malalignment. Little is known about the surgical strategies for these deformities, most especially the extent of fusion of vertebra involved.

Methods: We analyzed 24 patients with vertebral osteotomy at the level of the fracture and spinal fixation without pelvic fixation (local group), and 22 patients with vertebral osteotomy and pelvic fixation (pelvic group). Radiographic parameters, the incidence of proximal junctional kyphosis (PJK), distal junctional kyphosis (DJK), rod fractures, and the Oswestry Disability Index (ODI) were compared between the two groups over a 2-year follow-up period.

Results: In the pelvic group, postoperative spinopelvic parameters significantly improved, with the improvements maintained. No remarkable changes in spinopelvic parameters were seen in the local group. The mean ODI scores 2 years after surgery were 45.3 and 33.0 in the local and pelvic group, respectively $(p$-value $<0.05)$. There was no significant difference in the incidence of PJK in the local and pelvic groups, but there was a higher rate of DJK (41.7\%) in the local group. In contrast, rod fractures were more common in the pelvic group (45.5\%). Patients with DJK had higher ODI scores 2 years after surgery (52.0 in DJK patients vs. 34.8 in non-DJK patients; $p$-value $<0.05$ ).

Conclusions: For patients with rigid kyphosis due to osteoporotic vertebral fractures, better spinopelvic alignment and health-related quality of life can be achieved through extensive corrective surgery with pelvic fixation.

Keywords: Vertebral fracture; Kyphosis; Distal junctional kyphosis; Osteotomy; Surgery

Received Jan 13, 2020; Revised Mar 3, 2020; Accepted Mar 10, 2020

Corresponding author: Tomohiko Hasegawa

Department of Orthopedic Surgery, Hamamatsu University School of Medicine, 1-20-1 Handayama, Higashiku, Hamamatsu, Shizuoka, 431-3192, Japan

Tel: +81-53-435-2299, Fax: +81-53-435-2296, E-mail: hasetomo@hama-med.ac.jp 


\section{Introduction}

A study published in Japan in 2009 once reported that in a subset of people with an average age of 63 years, the prevalence rate of vertebral fractures was $22.6 \%$ [1]. In another investigation, the reported prevalence of vertebral fractures in patients between 70 to 75 years old was $25.0 \%$, and the prevalence rate increased to $43 \%$ for people aged over 80 years [2]. Ensrud et al. [3] conducted a survey of 6,439 elderly women and reported that the existence of thoracic vertebrae fractures was associated with spinal kyphosis. Osteoporotic vertebral fractures represent a significant portion of morbidity in the older age group, and are associated with eventual spinal kyphosis.

Osteoporotic vertebral fractures induce kyphosis, which remains even after synostosis of the fractures, contributing to abnormal posture [4], dysfunctional stride [5], easy falling syndrome [6], and fatigue-induced lumbago due to increased load on muscles, facets, and intervertebral discs. A study conducted by Schwab et al. reported that the health-related quality of life (HRQOL) of patients with spinal deformity was affected by sagittal spinopelvic parameters, such as pelvic incidence minus lumbar lordosis (PI-LL), sagittal vertical axis (SVA), and pelvic tilt (PT) [7]. Rigid kyphosis caused by vertebral fractures induces a variety of symptoms which may be considered as surgical indications for the correction of these deformities.

In our clinical experience in administering surgical treatment for such patients, those with rigid kyphosis due to an osteoporotic vertebral fracture show different clinical characteristics compared to middle-aged patients with traumatic vertebral fractures. Patients with rigid kyphosis show bone fragility, and tend to have more severe kyphosis and global spinopelvic malalignment compared to patients with traumatic vertebral fractures. These characteristics led us to two questions in decision-making for spinal deformity corrective surgery. The first question was, which surgery could improve the global malalignment and clinical outcomes-local or extensive corrective fixation surgery? The second question was whether the corrected alignment and clinical outcomes can be maintained or not. To our current knowledge, the answers to these questions are ambiguous.

The purpose of this study is to compare the spinopelvic parameters and clinical outcomes of local fixation to that of spinopelvic fixation for the target of treating rigid kyphosis due to osteoporotic vertebral fractures with spino- pelvic malalignment.

\section{Materials and Methods}

\section{Institutional review board approval}

This study was approved by the Institutional Review Board of Hamamatsu University School of Medicine Hospital (research approval no., 14-306). Informed consent was obtained from all individual participants including in the study.

\section{Study design and patients}

In this study, data were obtained for retrospective and prospective cohorts. We analyzed 52 consecutive patients who gave informed consent and underwent corrective surgery using vertebral osteotomies for rigid kyphosis induced by an osteoporotic vertebral fracture at Hamamatsu University School of Medicine Hospital between 2010 and 2015. The inclusion criteria were as follows: (1) cases aged $\geq 18$ years, (2) cases were followed-up for at least 2 years, (3) the presence of rigid kyphosis due to lower thoracic (T9 or below) or lumbar vertebral fractures, (4) cases with preoperative sagittal malalignment, and (5) those who were informed about the study and have consented to participate. Preoperative sagittal malalignment was defined as the presence of either SVA $\geq 50 \mathrm{~mm}, \mathrm{PI}-\mathrm{LL} \geq 10^{\circ}, \mathrm{PT} \geq 20^{\circ}$, or thoracic kyphosis (TK) $\geq 60^{\circ}$. Exclusion criteria were as follows: insufficient assessment of radiographs and HRQOL preoperatively, 6 months, and 2 years postoperatively. Out of 51 cases, five were excluded because they met this exclusion criterion. The remaining 46 cases were enrolled (mean age, 70.1 years; seven men and 39 women) (Table 1). The fixation level was determined according to active policies during the period wherein surgery was performed. Between January 2010 and April 2012, fixations were created at two or three segments above and below the fracture, using a vertebral osteotomy, without fixing the sacrum (local group). Between May 2012 and August 2015, the lowest instrumented vertebra was the pelvis using iliac screws, regardless of LL, local kyphosis angle, or level of vertebral osteotomy (pelvic group). Most of the patients had complete datasets that were sufficient for analysis. 
Table 1. Comparison of baseline and surgical data between the local and pelvic groups

\begin{tabular}{|c|c|c|c|}
\hline Characteristic & $\begin{array}{l}\text { Local group } \\
\qquad(\mathrm{n}=24)\end{array}$ & $\begin{array}{l}\text { Pelvic group } \\
\qquad(\mathrm{n}=22)\end{array}$ & $p$-value \\
\hline Age (yr) & $67.4 \pm 10.8$ & $70.2 \pm 6.9$ & 0.306 \\
\hline Female & $20(83.3)$ & $19(86.4)$ & 0.551 \\
\hline Height (cm) & $147.8 \pm 10.4$ & $149.8 \pm 9.6$ & 0.503 \\
\hline Body weight (kg) & $49.5 \pm 8.9$ & $54.1 \pm 13.8$ & 0.192 \\
\hline Body mass index $\left(\mathrm{kg} / \mathrm{m}^{2}\right)$ & $22.7 \pm 3.6$ & $24.1 \pm 6.1$ & 0.341 \\
\hline $\begin{array}{l}\text { Preoperative Oswestry Disability } \\
\text { Index score }\end{array}$ & $48.0 \pm 25.5$ & $45.6 \pm 16.1$ & 0.716 \\
\hline \multicolumn{4}{|l|}{ Surgical data } \\
\hline No. of fused vertebral segments & $8.5 \pm 1.0$ & $11.3 \pm 2.0$ & $0.005^{* *}$ \\
\hline Osteotomy level & $6.1 \pm 1.7$ & $4.4 \pm 1.4$ & $<0.001^{* *}$ \\
\hline No. of rods & & & 0.232 \\
\hline 2 & $21(87.5)$ & $15(68.2)$ & \\
\hline 3 & $2(8.3)$ & $3(13.6)$ & \\
\hline 4 & $1(4.2)$ & $4(18.2)$ & \\
\hline Length of surgery (min) & $329 \pm 53$ & $442 \pm 96$ & $<0.001^{* *}$ \\
\hline Estimated blood loss (mL) & $1,004 \pm 444$ & $2,166 \pm 1,114$ & $<0.001^{* *}$ \\
\hline
\end{tabular}

Values are presented as mean \pm standard deviation or number (\%).

${ }^{* *} p<0.01$; statistically significant difference.

\section{Selection of the level of vertebral osteotomy}

Patients with a fractured vertebral body having a posterior height over two-thirds of the vertebral body underwent corrective surgery with a pedicle subtraction osteotomy (PSO), as in the recommendations of Lehmer et al. [8]. Patients with a fractured vertebral body having a posterior height of less than two-thirds or those requiring local correction $\geq 35^{\circ}$ underwent a posterior vertebral column resection (PVCR).

\section{Radiographic evaluation, osteoporotic evaluation, and grouping}

Patient characteristics and surgical data of the sample population were initially obtained by review of medical records. Age, sex, height, body weight, body mass index (BMI), number of fused vertebral segments, osteotomy level, number of rods, length of surgery, and estimated blood loss were investigated. We defined the osteotomy levels as per the following rules: S1=0, L5 vertebral body $=1$, and $L 4$ vertebral body $=2 \ldots \mathrm{T} 1=17$. The measured radiographic parameters were T5-12 TK, L1-S1 LL, sacral slope, PT, PI, and SVA. Parameters were measured preoperatively, soon after surgery, and 2 years postoperatively. The following perioperative complications were also evaluated: postoperative motor deficit, delirium, surgical site infection, deep venous thrombosis, cardiac complications, pneumoniae, gastrointestinal complications, urinary tract infection, postoperative hematoma, proximal junctional kyphosis (PJK), proximal junctional failure (PJF), distal junctional kyphosis (DJK), distal junctional failure (DJF), and rod fractures. PJK was defined as a proximal junction angle (PJA) $\geq 20^{\circ}$ based on a study by Scheer et al. [9]. PJF was defined as a vertebral fracture of the upper instrumented vertebra (UIV), or an implant failure at the UIV and one or two vertebrae above. DJK was defined as a vertebral fracture, an implant failure, or a kyphosis angle $\geq 20^{\circ}$ on the caudal side. Clinical outcomes were assessed preoperatively, as well as 6 months, 1 year, and 2 years postoperatively using the Oswestry Disability Index (ODI) [10]. Furthermore, we performed a subgroup analysis of clinical outcomes based on the presence of postoperative complications.

\section{Statistical analysis}

Categorical variables were expressed as absolute numbers and percentages, and were analyzed by chi-square test or Fisher's exact test as appropriate. Shapiro-Wilk tests were used to determine whether continuous variables were normally or non-normally distributed. Continuous variables with normal distributions were expressed as means \pm standard deviations and were analyzed using unpaired $t$-tests. Furthermore, one-way analysis of variance and Kruskal-Wallis tests were used to analyze the change in ODI scores between the local and pelvic groups. Statistical analyses were conducted with IBM SPSS ver. 23.0 (IBM Corp., Armonk, NY, USA). All $p$-values $<0.05$ were considered statistically significant.

\section{Results}

The baseline characteristics, surgical data, and preoperative radiographic parameters in the local and pelvic groups are shown in Table 1 . There were 24 patients in the local group and 22 patients in the pelvic group. The age, sex, BMI, preoperative ODI scores, and number of rods did not differ significantly between the local and pelvic groups. The osteotomy levels were at $6.1 \pm 1.7$ and $4.4 \pm 1.4$ in the local and global groups, respectively ( $p$-value 
Table 2. Comparison of radiographic parameters between the local and pelvic groups

\begin{tabular}{|c|c|c|c|}
\hline Variable & $\begin{array}{l}\text { Local group } \\
\qquad(\mathrm{n}=24)\end{array}$ & $\begin{array}{l}\text { Pelvic group } \\
\qquad(\mathrm{n}=22)\end{array}$ & $p$-value \\
\hline \multicolumn{4}{|l|}{ Preoperatively } \\
\hline Thoracic kyphosis $\left({ }^{\circ}\right)$ & $51.5 \pm 21.7$ & $31.4 \pm 19.2$ & $0.002^{* *}$ \\
\hline Lumbar lordosis $\left({ }^{\circ}\right)$ & $36.6 \pm 27.0$ & $11.3 \pm 30.7$ & $0.005^{* *}$ \\
\hline Sagittal vertical axis (mm) & $70.7 \pm 72.5$ & $138.7 \pm 99.5$ & $0.012^{* *}$ \\
\hline Pelvic tilt $\left({ }^{\circ}\right)$ & $30.7 \pm 13.1$ & $36.1 \pm 14.2$ & 0.185 \\
\hline Sacral slope $\left({ }^{\circ}\right)$ & $22.5 \pm 13.3$ & $18.0 \pm 14.5$ & 0.278 \\
\hline Pelvic incidence $\left({ }^{\circ}\right)$ & $53.2 \pm 13.7$ & $54.1 \pm 11.7$ & 0.808 \\
\hline PI-LL ( $\left.{ }^{\circ}\right)$ & $16.6 \pm 24.9$ & $42.8 \pm 29.8$ & $0.002^{* *}$ \\
\hline \multicolumn{4}{|l|}{ Soon after operation } \\
\hline Thoracic kyphosis $\left({ }^{\circ}\right)$ & $39.8 \pm 10.9$ & $35.3 \pm 10.2$ & 0.16 \\
\hline Lumbar lordosis $\left({ }^{\circ}\right)$ & $32.7 \pm 19.4$ & $43.1 \pm 9.9$ & $0.030^{*}$ \\
\hline Sagittal vertical axis (mm) & $63.1 \pm 44.6$ & $52.4 \pm 58.1$ & 0.492 \\
\hline Pelvic tilt $\left({ }^{\circ}\right)$ & $30.3 \pm 10.0$ & $21.0 \pm 9.5$ & $0.003^{* *}$ \\
\hline Sacral slope $\left({ }^{\circ}\right)$ & $23.0 \pm 10.6$ & $34.0 \pm 9.8$ & $0.001 * *$ \\
\hline PI-LL ( $\left.{ }^{\circ}\right)$ & $20.5 \pm 16.9$ & $11.0 \pm 14.3$ & $0.049^{*}$ \\
\hline \multicolumn{4}{|c|}{$\begin{array}{c}\text { Change of parameters (soon after } \\
\text { operation-preoperatively) }\end{array}$} \\
\hline Thoracic kyphosis $\left({ }^{\circ}\right)$ & $-13.2 \pm 14.4$ & $3.9 \pm 18.5$ & $0.001^{* *}$ \\
\hline Lumbar lordosis $\left({ }^{\circ}\right)$ & $-3.7 \pm 18.5$ & $31.8 \pm 29.4$ & $<0.001^{* *}$ \\
\hline Sagittal vertical axis $(\mathrm{mm})$ & $-7.6 \pm 52.0$ & $-86.3 \pm 93.1$ & $0.001^{* *}$ \\
\hline Pelvic tilt $\left({ }^{\circ}\right)$ & $-1.6 \pm 6.6$ & $-15.2 \pm 15.6$ & $<0.001^{* *}$ \\
\hline Sacral slope $\left({ }^{\circ}\right)$ & $1.7 \pm 6.1$ & $16.0 \pm 13.7$ & $<0.001^{* *}$ \\
\hline \multicolumn{4}{|l|}{ Two years postoperatively } \\
\hline Thoracic kyphosis $\left({ }^{\circ}\right)$ & $44.9 \pm 16.5$ & $44.8 \pm 10.9$ & 0.988 \\
\hline Lumbar lordosis $\left({ }^{\circ}\right)$ & $25.4 \pm 31.4$ & $40.3 \pm 13.2$ & 0.062 \\
\hline Sagittal vertical axis (mm) & $102.0 \pm 79.8$ & $71.6 \pm 59.6$ & 0.196 \\
\hline Pelvic tilt $\left({ }^{\circ}\right)$ & $32.8 \pm 15.0$ & $28.3 \pm 11.0$ & 0.301 \\
\hline Sacral slope $\left({ }^{\circ}\right)$ & $21.2 \pm 14.1$ & $27.7 \pm 11.1$ & 0.127 \\
\hline $\mathrm{PI}-\mathrm{LL}\left({ }^{\circ}\right)$ & $29.8 \pm 28.5$ & $11.9 \pm 16.4$ & $0.02^{*}$ \\
\hline
\end{tabular}

Values are presented as mean \pm standard deviation or number $(\%)$.

$\mathrm{PI}-\mathrm{LL}$, pelvic incidence minus lumbar lordosis $\left({ }^{\circ}\right)$.

${ }^{*} p<0.05$ and ${ }^{* *} p<0.01$; statistically significant difference.

$<0.001$ ), with the osteotomy levels significantly higher in the local group. Compared with the local group, the pelvic group had a significantly larger number of fused vertebral segments, longer length of surgery, and greater estimated blood loss ( $p=0.005, p<0.001$, and $p<0.001$, respectively).

\section{Changes in spinopelvic parameters}

The radiographic data of both groups are shown in Table
Table 3. Incidence of perioperative complications and revision surgeries between the local and pelvic groups

$\begin{array}{ccc}\text { Vocal group } & \text { Pelvic group } \\ (\mathrm{n}=24) & (\mathrm{n}=22) & p \text {-value }\end{array}$

Perioperative complication

\begin{tabular}{|lccc|}
\hline Postoperative motor deficit & 0 & $2(9.1)$ & 0.149 \\
\hline Delirium & $3(12.5)$ & $2(9.1)$ & 0.711 \\
\hline Surgical site infection & $1(4.2)$ & $1(4.5)$ & 0.950 \\
\hline Deep venous thrombosis & 0 & $1(4.5)$ & 0.312 \\
\hline Cardiac complications & 0 & $1(4.5)$ & 0.312 \\
\hline Pneumoniae & 0 & $1(4.5)$ & 0.312 \\
\hline Gastrointestinal complications & $1(4.2)$ & $3(13.6)$ & 0.255 \\
\hline Urinary tract infection & $2(8.3)$ & 0 & 0.166 \\
\hline Postoperative hematoma & 0 & $1(4.5)$ & 0.312 \\
\hline
\end{tabular}

Instrumentation failure

\begin{tabular}{lccc}
$\begin{array}{l}\text { Proximal junctional kyphosis or } \\
\text { failure }\end{array}$ & $4(16.7)$ & $7(31.8)$ & 0.229 \\
$\begin{array}{l}\text { Distal junctional kyphosis or } \\
\text { failure }\end{array}$ & $10(41.7)$ & $1(4.5)$ & $0.003^{* *}$ \\
\hline $\begin{array}{l}\text { Rod fractures } \\
\text { Group with proximal junctional } \\
\text { kyphosis }\end{array}$ & 0 & $14(63.6)$ & $<0.001^{* *}$ \\
\hline $\begin{array}{l}\text { Total no. of patients } \\
\text { Presence of revision surgery }\end{array}$ & 0 & $1(14.3)$ & 0.460 \\
\hline $\begin{array}{l}\text { Group with distal junctional } \\
\text { kyphosis }\end{array}$ & & & \\
\hline $\begin{array}{l}\text { Total no. of patients } \\
\text { Presence of revision surgery }\end{array}$ & $4(40.0)$ & 0 & 0.428 \\
\hline $\begin{array}{l}\text { Group with rod fracture } \\
\text { Total no. of patients }\end{array}$ & 0 & 14 & \\
\hline Presence of revision surgery & 0 & $14(100.0)$ & - \\
\hline
\end{tabular}

Values are presented as number (\%).

${ }^{* *} p<0.01$; statistically significant difference.

2. The preoperative TK and LL were lower in the pelvic group than in the local group ( $p=0.002$ and $p=0.005$, respectively). The preoperative SVA and PI-LL were significantly higher in the pelvic group than in the local group ( $p=0.012$ and $p=0.002$, respectively). In the pelvic group, the postoperative SVA showed a larger decrease, while the postoperative LL showed a larger increase compared to the local group due to deformity correction, and the improvements were maintained for 2 years. Two years after surgery, PI-LL was significantly higher in the local group $\left(29.8^{\circ}\right)$ than in the pelvic group $\left(11.9^{\circ}\right)(p=0.020)$. 
Table 4. Pre- and postoperative ODI scores between both groups related postoperative complication

\begin{tabular}{|c|c|c|c|c|c|c|c|c|c|}
\hline ODI & $\begin{array}{l}\text { Non-PJK } \\
\text { group }(n=35)\end{array}$ & $\begin{array}{l}\text { PJK group } \\
(n=11)\end{array}$ & $p$-value & $\begin{array}{c}\text { Non-DJK } \\
\text { group }(n=35)\end{array}$ & $\begin{array}{l}\text { DJK group } \\
(n=11)\end{array}$ & $p$-value & $\begin{array}{c}\text { Non-rod } \\
\text { fracture }(n=36)\end{array}$ & $\begin{array}{l}\text { Rod fracture } \\
\qquad(\mathrm{n}=10)\end{array}$ & $p$-value \\
\hline Preop score & $46.3 \pm 22.6$ & $48.4 \pm 17.1$ & 0.785 & $45.0 \pm 19.5$ & $52.5 \pm 26.1$ & 0.309 & $45.5 \pm 22.2$ & $51.6 \pm 17.3$ & 0.426 \\
\hline Postop score (6 mo postop) & $35.8 \pm 21.3$ & $35.9 \pm 15.9$ & 0.990 & $31.9 \pm 18.2$ & $47.7 \pm 20.8$ & $0.020^{*}$ & $38.1 \pm 19.8$ & $28.3 \pm 19.4$ & 0.176 \\
\hline Postop score (1 yr postop) & $33.8 \pm 19.5$ & $37.9 \pm 18.3$ & 0.539 & $31.5 \pm 17.8$ & $44.8 \pm 20.1$ & $0.043^{*}$ & $37.0 \pm 19.0$ & $27.3 \pm 18.5$ & 0.161 \\
\hline Postop score (2 yr postop) & $40.2 \pm 24.3$ & $37.4 \pm 19.2$ & 0.743 & $34.8 \pm 19.3$ & $52.0 \pm 27.8$ & $0.032^{*}$ & $41.6 \pm 23.0$ & $33.3 \pm 22.7$ & 0.330 \\
\hline
\end{tabular}

Values are presented as mean \pm standard deviation.

ODI, Oswestry Disability Index; PJK, proximal junctional kyphosis; DJK, distal junctional kyphosis; Preop, preoperative; Postop, postoperative.

${ }^{*} p<0.05$; statistically significant difference.

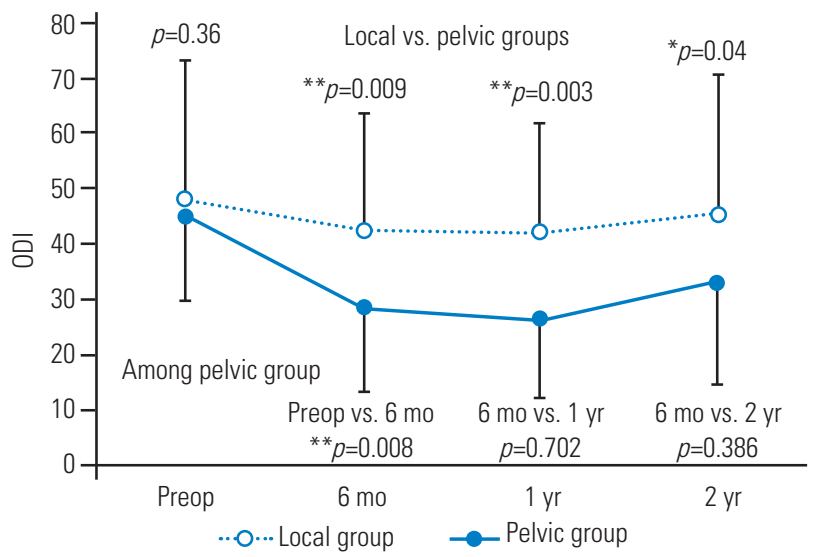

Fig. 1. Change of Oswestry Disability Index (ODI) score between local and pelvic groups. Preop, preoperative. ${ }^{*} p<0.05 .{ }^{* *} p<0.01$.

\section{Postoperative complications and clinical outcomes}

The incidence of perioperative complications in the local and pelvic groups is shown in Table 3. Either PJK or PJF occurred in four patients (16.7\%) in the local group and seven patients (31.8\%) in the pelvic group ( $p=0.229)$. DJK or DJF occurred in 10 patients (41.7\%) in the local group and one patient $(4.5 \%)$ in the pelvic group $(p=0.003)$. Rod fractures occurred in 14 patients $(63.6 \%)$ in the pelvic group, and no rod fractures occurred in the local group $(p<0.001)$. There was no significant difference in the incidence of other perioperative complications between local and pelvic groups.

The incidence of revision surgeries in the local and pelvic groups is shown in Table 3. A revision surgery after PJF occurred in one patient (14.3\%) in the pelvic group, while no revision surgeries after PJF occurred in the local group ( $p=0.460)$. A revision surgery after DJF was done in four patients $(40.0 \%)$ in the local group, and no revision surgeries after DJF occurred in the pelvic group ( $p=0.428$ ). The revision surgery after rod fractures occurred in $14 \mathrm{pa}-$ tients (100\%) in the pelvic group.

Changes in the ODI scores in the local and pelvic groups are shown in Fig. 1. ODI scores in the local and pelvic groups were $42.5 \pm 21.5$ and $28.6 \pm 15.5$, respectively, 6 months after surgery ( $p=0.009), 42.2 \pm 20.0$ and $26.7 \pm 14.5$, respectively 1 year after surgery $(p=0.003)$, and $45.3 \pm 25.5$ and $33.0 \pm 18.1$, respectively 2 years after surgery $(p=0.040)$. In the local group, there was no significant difference between preoperative and postoperative ODI scores. In contrast, in the pelvic group, preoperative ODI scores $(45.6 \pm 16.1)$ improved significantly to $28.6 \pm 15.5$ points 6 months after surgery $(p=0.008)$, and postoperative ODI scores were maintained after 1 year $(26.7 \pm 14.5$ versus 6 months postoperatively, $p=0.702)$ and after 2 years $(33.0 \pm 18.1$ versus 6 months postoperatively, $p=0.386$ ).

\section{Subgroup analysis of clinical outcomes}

Preoperative ODI scores were not significantly different compared to the 6-month, 1-year, and 2-year postoperative scores in the PJK and non-PJK groups ( $p=0.785$, $p=0.990, p=0.539$, and $p=0.743$, respectively). However, the DJK group showed significantly higher ODI scores than the non-DJK group at 6 months, 1 year, and 2 years after surgery ( $p=0.020, p=0.043$, and $p=0.032$, respectively). Preoperative, 6-month, 1-year, and 2-year postoperative ODI scores did not differ significantly in patients with rod fractures and those without $(p=0.426, p=0.176$, $p=0.161$, and $p=0.330$, respectively) (Table 4 ).

\section{Case presentation}

Patient 1 was a 68-year-old female with back pain and rigid kyphosis due to T11 vertebral fracture with mild sagittal malalignment (Fig. 2). Local corrective surgery re- 


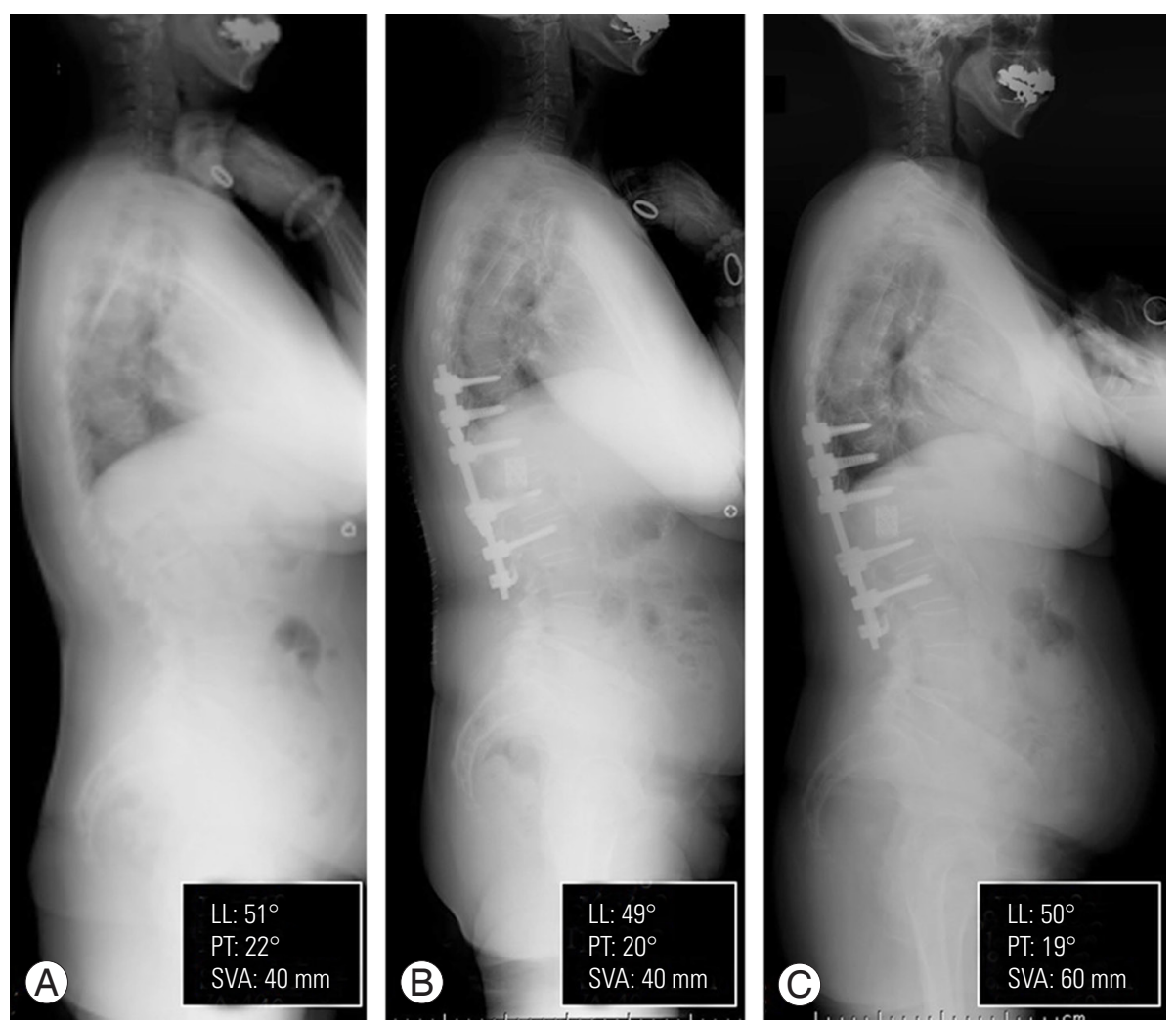

Fig. 2. Radiographs of a successful case managed through local correction and fixation. (A) Case 1: a 68-year-old female with back pain and rigid kyphosis due to a T11 vertebral fracture with mild sagittal malalignment. (B) The patient underwent T11 posterior vertebral column resection and posterior fixation from T8 to L1. (C) Radiograph at 3 years after surgery showed maintenance of good sagittal alignment. Her back pain resolved postoperatively. LL, lumbar lordosis; PT, pelvic tilt; SVA, sagittal vertical axis.

sulted in good clinical outcomes. Patient 2 was a 74-yearold male with back pain and severe kyphosis due to T10 vertebral fracture with severe global malalignment. PJF and DJF occurred 4 months after local corrective surgery due to T7 and L4 vertebral fractures. The patient underwent reoperation with additional L4 PSO and long fusion to the pelvis (Figs. 3, 4).

\section{Discussion}

This study showed that in surgical treatments for rigid kyphosis due to osteoporotic vertebral fractures with sagittal malalignment, local corrective surgery without pelvic fixation did not improve sagittal global malalignment and HRQOL postoperatively. Better spinopelvic alignment and HRQOL were achieved with extensive corrective surgery with pelvic fixation. As for postoperative complications, DJK occurred in about $42 \%$ of patients after local corrective surgery, and rod fractures occurred in about $64 \%$ of patients after extensive corrective surgery. Given that patients with DJK after local corrective surgery without pelvic fixation did not show improvement and maintenance of HRQOL, extensive corrective surgery with pelvic fixation might be a better option for corrective surgery in patients with rigid kyphosis due to osteoporosis vertebral fractures.

Crawford et al. [11] reported that extensive corrective surgery with pelvic fixation for patients aged 65 years or older with spinal deformities was effective for maintaining good outcomes until the 2-year follow-up, and this result was comparable to results achieved following surgery on patients aged 55 years or younger. However, extensive corrective surgery with vertebral osteotomy was associated with several serious problems in this study, including longer surgery duration (442 minutes) and greater estimated blood loss (2,166 mL). Yoshida et al. [12] defined a length of surgery $\geq 6$ hours or estimated blood loss $\geq 2,000 \mathrm{~mL}$ as risk factors triggering various postoperative complications in patients with adult spinal deformities. In future studies, the reduction of surgical invasiveness and further 

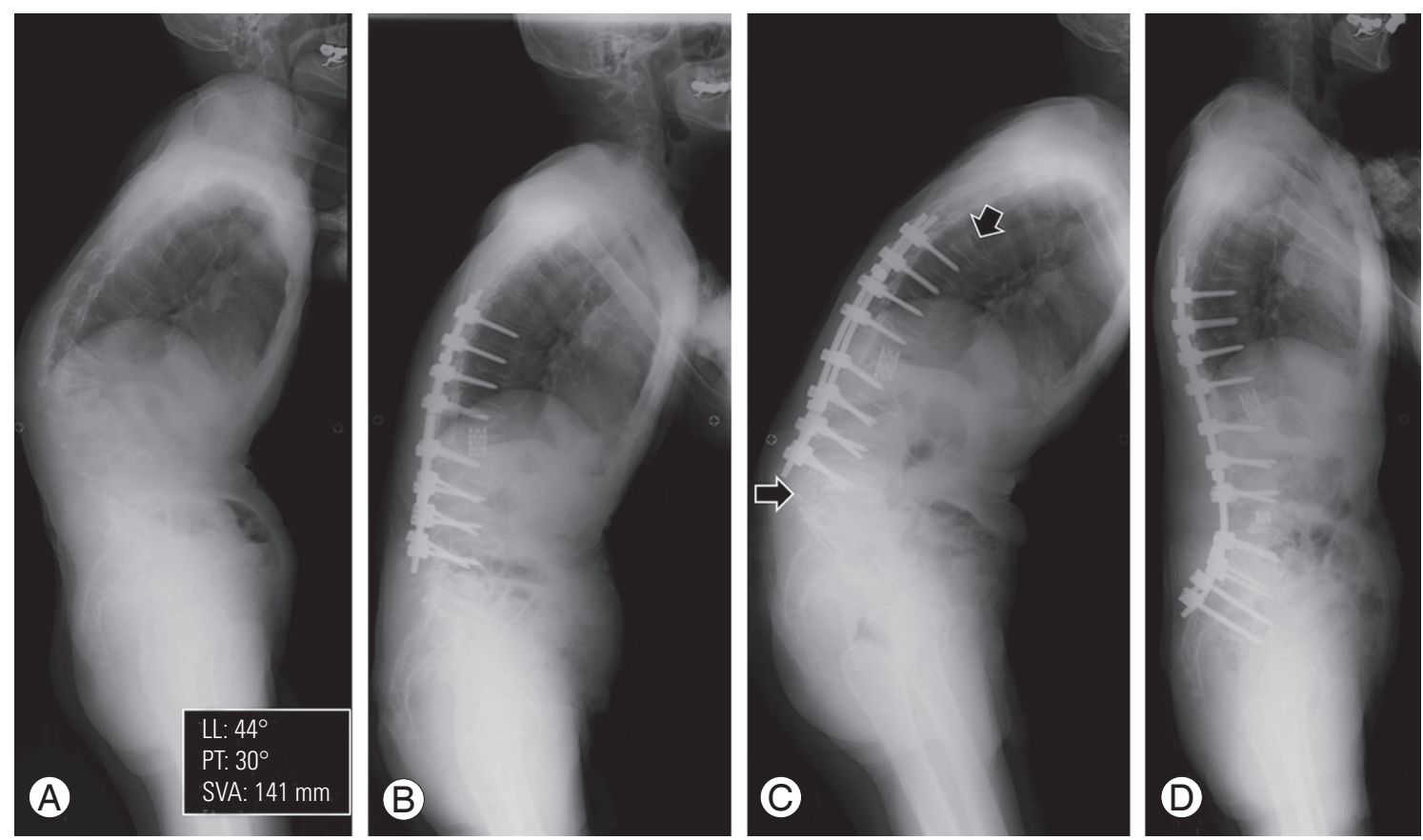

Fig. 3. Radiographs of an unsuccessful case resulting in distal junctional kyphosis after local correction. (A) Case 2: a 74-year-old male with back pain and severe kyphosis due to a T10 vertebral fracture. (B) The patient underwent T10 posterior vertebral column resection and posterior fixation from T8 to L4. (C) T7 and L4 vertebral fractures occurred 4 months after surgery (arrows). (D) L4 pedicle subtraction osteotomy and spinopelvic fixation were performed. The radiograph taken 2 years after surgery showed maintenance of good sagittal alignment. LL, lumbar lordosis; PT, pelvic tilt; SVA, sagittal vertical axis.
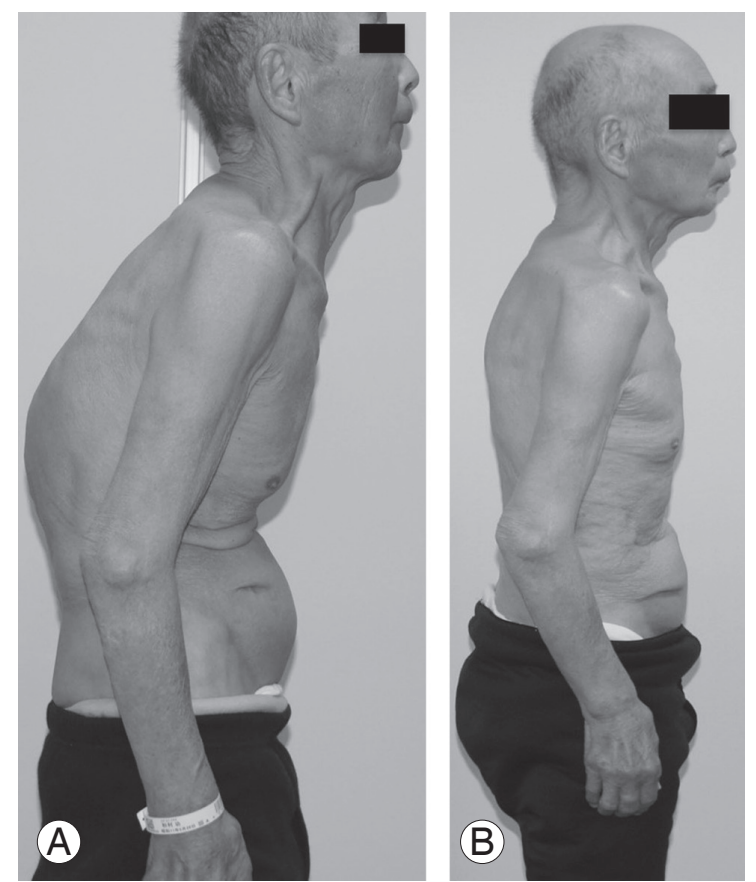

Fig. 4. Standing photographs of case 2 preoperatively and 2 years after operation. (A) Preoperative lateral photograph revealed severe kyphosis of the thoracolumbar junctional level. The patient had difficulty standing upright. (B) Lateral photograph 2 years after $L 4$ pedicle subtraction osteotomy and spinopelvic fixation revealed resolution of severe kyphosis. The patient could stand upright. The patient provided written informed consent for publication of clinical details and images. development of surgical procedures will be important for perioperative safety.

Another previous report showed that extensive corrective surgery including pelvic fixation was associated with the development of PJK in adults with spinal deformity [13]. Furthermore, aging, previous spinal surgery, and UIV level at the thoracolumbar junction were also associated with PJK [14]. In this study, there was no significant difference in the incidence of PJK or PJF between patients with and without pelvic fixation. Kwon et al. [15] reported that the development of DJK was related to osteoporosis, previous total hip arthroplasty, and substance abuse. While this study did not include patients with previous total hip arthroplasty or substance abuse, all patients were elderly and were predisposed to osteoporosis, which might have led to the higher incidence of DJK. These results showed that both the local and pelvic groups had continuous burden on the trunk despite corrective surgery, which focused on either the upper or lower part of fixation according to the range of fused vertebral segments.

Most of the previous studies have defined PJK as a PJA at least $10^{\circ}$ greater than the preoperative measurement 
$[13,14,16,17]$. Some studies have reported that a cut-off value of $10^{\circ}$ was not associated with any clinical symptoms, and that a cut-off value of $20^{\circ}$ was more appropriate $[9,18]$. In this study, PJK was defined as a PJA of least $20^{\circ}$ greater than the preoperative measurement. Results showed that there was no significant difference in ODI scores 2 years after surgery between patients with PJK and those without. In contrast, ODI deteriorated significantly more in patients with DJK compared with those without, and the improvement of HRQOL was poorer in elderly patients with DJK compared to those with PJK. A previous report showed that ODI was associated with an increase in PI-LL, PT, and SVA [7]. Janik et al. [19] reported that the L3 to S1 formed 85\% of LL, so the progress of lumbar kyphosis on the middle and lower lumbar level may have influenced a reduction in LL and an eventual deterioration in spinopelvic global alignment. Patients with DJK generally showed reduction of LL due to a distal vertebral fracture at the middle or lower lumbar level because of instrumentation at the lower vertebra. In this study, the reduction of LL occurred with deterioration in SVA and PI-LL in patients who underwent local corrective surgery.

Patients who underwent extensive corrective surgery including spinopelvic fixation showed improvement and maintenance of spinopelvic malalignment and HRQOL. However, extensive corrective surgery was also associated with a higher incidence of rod fractures compared to local corrective surgery without pelvic fixation. Furthermore, since rod fractures often occurred at the level of the osteotomy, all cases with rod fractures required revision surgery. Nevertheless, after undergoing revision surgery, the improvement of HRQOL was maintained.

Outcomes of a basic experiment reported that the combination of satellite rods reduced the stress on rods by $59 \%$ [20]. It was reported that usage of satellite rods in patients with Scheuermann kyphosis resulted in greater correction and maintenance of the correction [21]. Satellite rods covering the osteotomy site and lumbosacral junction reduced the incidence of rod fractures following PSO or PVCR surgery with pelvic fixation [22]. Given that evidence suggests that incorporating the satellite rod technique can reduce the incidence of rod fractures, it is necessary to consider the combination of satellite rods with extensive corrective surgery and vertebral osteotomy in future studies on patients with osteoporosis.

This study had several limitations. First, it was a small, retrospective study from a prospectively corrected database which limits the generalizability and causality of the results. Second, this study included mostly elderly patients with rigid kyphosis due to osteoporotic vertebral fractures, and patients in the local group had osteotomy levels that were more proximal than those of the patients in the spinopelvic fixation group. Furthermore, patients in the spinopelvic fixation group had significant greater severities of sagittal malalignment and PI-LL mismatch preoperatively, than the patients in the local group. However, even under these conditions, this study found that extensive corrective surgery with vertebral osteotomy was superior to local corrective surgery, both in terms of improvement and maintenance of sagittal malalignment and HRQOL. Third, the postoperative observation period of 2 years was relatively short, so future studies with a longer follow-up period of five to 10 years are needed to evaluate long-term outcomes.

\section{Conclusions}

In surgical treatments for rigid kyphosis due to osteoporotic vertebral fracture, local corrective surgery without pelvic fixation did not improve sagittal global malalignment and HRQOL. On the other hand, better spinopelvic alignment and HRQOL were achieved using extensive corrective surgery with pelvic fixation in patients with kyphosis. Extensive corrective surgery may be a better option for corrective surgery of rigid kyphosis in avoiding DJK, as DJK may induce postoperative deterioration of HRQOL.

\section{Conflict of Interest}

No potential conflict of interest relevant to this article was reported.

\section{Acknowledgments}

We appreciate the help of Daisuke Togawa, Sho Kobayashi, and Yuki Mihara for case collections or manuscript discussions.

\section{Author Contributions}

Yukihiro Matsuyama supervised the study. Tomohiko Hasegawa was responsible for the study's conception and 
design. Tomohiko Hasegawa and Hiroki Ushirozako acquired, analyzed, and interpreted data, drafted the article, and approved the final version on behalf of all authors. All authors have critically revised the article and reviewed the submitted version.

\section{References}

1. Yoshimura N, Muraki S, Oka H, et al. Prevalence of knee osteoarthritis, lumbar spondylosis, and osteoporosis in Japanese men and women: the research on osteoarthritis/osteoporosis against disability study. J Bone Miner Metab 2009;27:620-8.

2. Ross PD, Fujiwara S, Huang C, et al. Vertebral fracture prevalence in women in Hiroshima compared to Caucasians or Japanese in the US. Int J Epidemiol 1995;24:1171-7.

3. Ensrud KE, Thompson DE, Cauley JA, et al. Prevalent vertebral deformities predict mortality and hospitalization in older women with low bone mass: Fracture Intervention Trial Research Group. J Am Geriatr Soc 2000;48:241-9.

4. Kim HJ, Yi JM, Cho HG, et al. Comparative study of the treatment outcomes of osteoporotic compression fractures without neurologic injury using a rigid brace, a soft brace, and no brace: a prospective randomized controlled non-inferiority trial. J Bone Joint Surg Am 2014;96:1959-66.

5. Katzman WB, Vittinghoff E, Ensrud K, Black DM, Kado DM. Increasing kyphosis predicts worsening mobility in older community-dwelling women: a prospective cohort study. J Am Geriatr Soc 2011;59:96100.

6. Van der Jagt-Willems HC, de Groot $\mathrm{MH}$, van Campen JP, Lamoth CJ, Lems WF. Associations between vertebral fractures, increased thoracic kyphosis, a flexed posture and falls in older adults: a prospective cohort study. BMC Geriatr 2015;15:34.

7. Schwab F, Ungar B, Blondel B, et al. Scoliosis Research Society-Schwab adult spinal deformity classification: a validation study. Spine (Phila Pa 1976) 2012;37:1077-82.

8. Lehmer SM, Keppler L, Biscup RS, Enker P, Miller $\mathrm{SD}$, Steffee AD. Posterior transvertebral osteotomy for adult thoracolumbar kyphosis. Spine (Phila Pa 1976) 1994;19:2060-7.

9. Scheer JK, Fakurnejad S, Lau D, et al. Results of the
2014 SRS survey on PJK/PJF: a report on variation of select SRS member practice patterns, treatment indications, and opinions on classification development. Spine (Phila Pa 1976) 2015;40:829-40.

10. Fairbank JC, Pynsent PB. The Oswestry Disability Index. Spine (Phila Pa 1976) 2000;25:2940-52.

11. Crawford CH 3rd, Carreon LY, Bridwell KH, Glassman SD. Long fusions to the sacrum in elderly patients with spinal deformity. Eur Spine J 2012;21:2165-9.

12. Yoshida G, Hasegawa T, Yamato Y, et al. Predicting perioperative complications in adult spinal deformity surgery using a simple sliding scale. Spine (Phila Pa 1976) 2018;43:562-70.

13. Maruo K, Ha Y, Inoue $S$, et al. Predictive factors for proximal junctional kyphosis in long fusions to the sacrum in adult spinal deformity. Spine (Phila $\mathrm{Pa}$ 1976) 2013;38:E1469-76.

14. Yagi M, Rahm M, Gaines R, et al. Characterization and surgical outcomes of proximal junctional failure in surgically treated patients with adult spinal deformity. Spine (Phila Pa 1976) 2014;39:E607-14.

15. Kwon BK, Elgafy H, Keynan O, et al. Progressive junctional kyphosis at the caudal end of lumbar instrumented fusion: etiology, predictors, and treatment. Spine (Phila Pa 1976) 2006;31:1943-51.

16. Glattes RC, Bridwell KH, Lenke LG, Kim YJ, Rinella A, Edwards C 2nd. Proximal junctional kyphosis in adult spinal deformity following long instrumented posterior spinal fusion: incidence, outcomes, and risk factor analysis. Spine (Phila Pa 1976) 2005;30:1643-9.

17. Yagi $M$, King $A B$, Boachie-Adjei O. Incidence, risk factors, and natural course of proximal junctional kyphosis: surgical outcomes review of adult idiopathic scoliosis: minimum 5 years of follow-up. Spine (Phila Pa 1976) 2012;37:1479-89.

18. Oe S, Togawa D, Hasegawa T, et al. The risk of proximal junctional kyphosis decreases in patients with optimal thoracic kyphosis. Spine Deform 2019;7:75970.

19. Janik TJ, Harrison DD, Cailliet R, Troyanovich SJ, Harrison DE. Can the sagittal lumbar curvature be closely approximated by an ellipse? J Orthop Res 1998;16:766-70.

20. Januszewski J, Beckman JM, Harris JE, Turner AW, Yen CP, Uribe JS. Biomechanical study of rod stress after pedicle subtraction osteotomy versus anterior 
column reconstruction: a finite element study. Surg Neurol Int 2017;8:207.

21. Zhu ZZ, Chen X, Qiu Y, et al. Adding satellite rods to standard two-rod construct with the use of duet screws: an effective technique to improve surgical outcomes and preventing proximal junctional ky- phosis in posterior-only correction of Scheuermann kyphosis. Spine (Phila Pa 1976) 2018;43:E758-65.

22. Yamato Y, Hasegawa T, Togawa D, et al. Long additional rod constructs can reduce the incidence of rod fractures following 3-column osteotomy with pelvic fixation in short term. Spine Deform 2020;8:481-90. 